Revue des patrimoines

16 | 2011

Le patrimoine militaire et la question urbaine

\title{
Le front oriental de Lille
}

\section{Étienne Poncelet}

\section{OpenEdition}

Journals

Édition électronique

URL : http://journals.openedition.org/insitu/197

DOI : 10.4000/insitu.197

ISSN : 1630-7305

\section{Éditeur}

Ministère de la culture

\section{Référence électronique}

Étienne Poncelet, « Le front oriental de Lille », In Situ [En ligne], 16 | 2011, mis en ligne le 20 juin 2011 , consulté le 19 avril 2019. URL : http://journals.openedition.org/insitu/197 ; DOI : 10.4000/insitu.197

Ce document a été généré automatiquement le 19 avril 2019

\section{(2) $\odot \Theta \Theta$}

In Situ Revues des patrimoines est mis à disposition selon les termes de la licence Creative Commons Attribution - Pas d'Utilisation Commerciale - Pas de Modification 4.0 International. 


\title{
Le front oriental de Lille
}

\author{
Étienne Poncelet
}

1 De la porte d'eau de la Basse Deûle jusqu'au fort Saint-Sauveur, le front oriental de Lille, fortifié à l'époque espagnole, glisse ses courtines dans les entrelacs du boulevard périphérique et des gares. L'enjeu urbain actuel consiste à s'appuyer sur ces murs historiques pour " passer malgré tout » à travers cet écheveau urbain et retisser les fils de la continuité des promenades au cœur de la ville. Moins connus que le front occidental de la reine des citadelles, ces anciens espaces militaires sont une chance pour l'urbanisme de demain dont les opérations en cours de la Porte de Gand et de la Basse Deûle témoignent déjà.

2 Épargnée par l'extension de la ville en 1865 vers le sud et l'ouest, cette ligne fortifiée n'a été que partiellement démantelée - si l'on excepte les démolitions des années 1990 pour ouvrir le quartier d'Euralille, malgré les propositions de «dialogue fortifié » que nous avions faites à l'époque à Rem Koolhaas, l'auteur du « bigness ».

3 Ce front oriental égrène encore une série de sites fortifiés remarquables, heureusement pris en compte par la municipalité actuelle comme un atout du développement de la ville. Ce front représente toute la partie du Lille ancien située à l'est de la "capitale " principale de la ville, au sens militaire du terme.

4 Le Lille ancien est en effet traversé par une grande ligne nord-sud qui relie la porte d'eau de la Basse Deûle au fort Saint-Sauveur dirigé vers la France, en alignant les points remarquables du dôme de la Madeleine, de l'ancien château de Courtrai et du chevet de l'antique église Saint-Maurice (fig. $\left.\mathbf{n}^{\mathbf{0}} \mathbf{1}\right)$. 


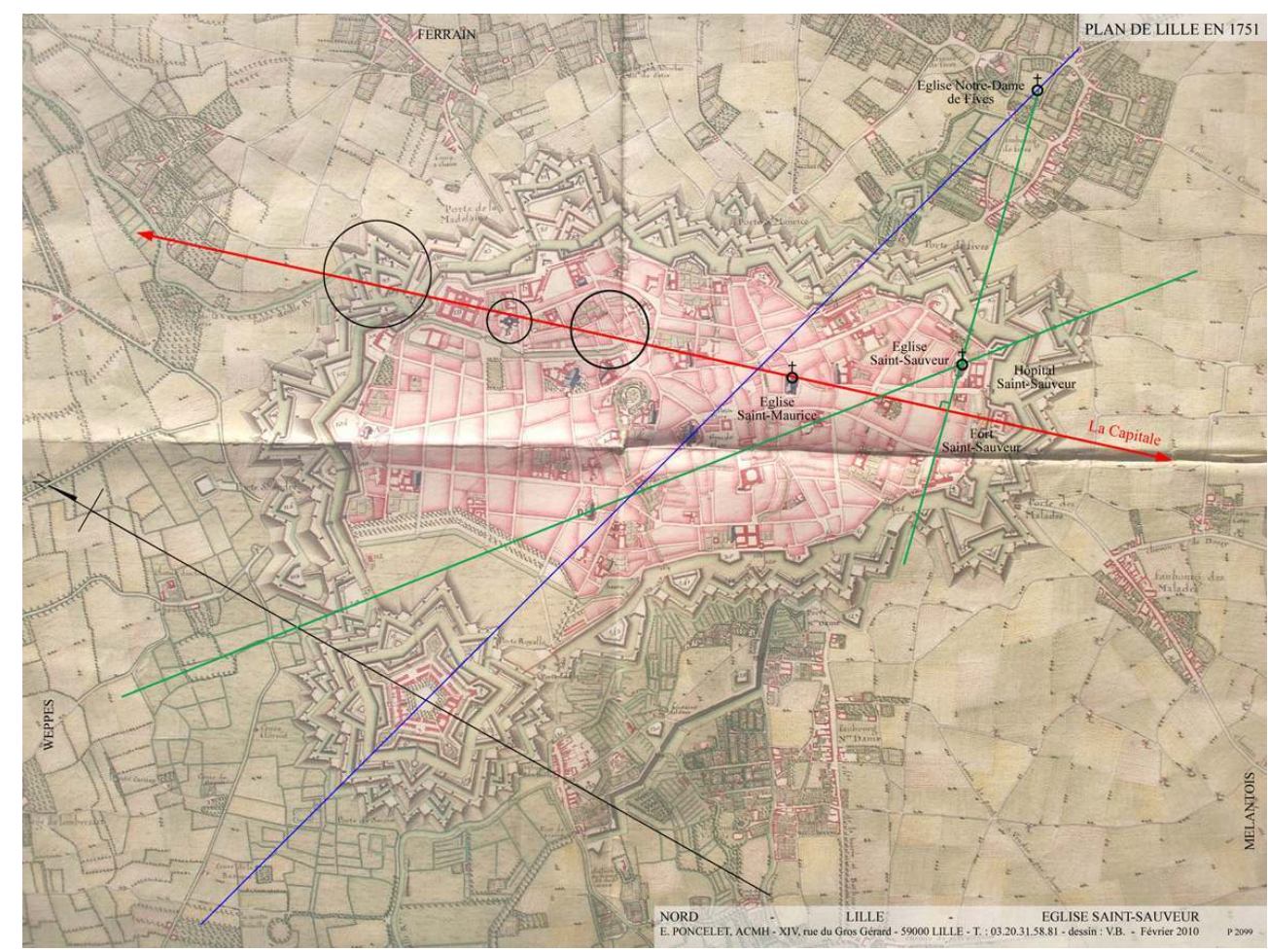

Le front oriental de Lille, à l'est de la grande capitale reliant la porte d'eau de la Basse Deûle au nord et le fort Saint-Sauveur au sud.

Repro. E. Poncelet.

5 À l'est de cette ligne, une mince bande d'urbanisation se resserre contre le front oriental de la ville, correspondant à l'extension de l'époque des archiducs et du quartier SaintSauveur.

6 Adossé au versant géologique qui marque le début du pays de Ferrain, ce front oriental de la ville est une limite urbaine d'une remarquable permanence. La ville ne s'est jamais développée au-delà de cette ligne, parcourue aujourd'hui par le boulevard périphérique et la ligne TGV. Le projet d'Euralille, à cheval sur la gare, tente, en vain, d'établir une passerelle sur ce front naturel et historique qui résiste avec une étonnante force du lieu.

7 Garanti par cette légitimité géohistorique, le front oriental de Lille a conservé les points remarquables de l'histoire de son enceinte militaire, depuis la porte d'eau, les portes espagnoles de Gand et de Roubaix jusqu'à la Noble Tour, de l'époque bourguignonne.

Malgré les renforts de Vauban, en avant de ces différents ouvrages, notamment le grand ouvrage à cornes de la porte de la Madeleine, la physionomie de ce front reste, pour l'essentiel, celle de l'enceinte de la ville avant la période française.

Intra muros, ce rempart abrite les grands ensembles institutionnels : l'hôpital général (1739-1836), les casernes du quartier de la Corne de Gand dont l'État-Major actuel de la Région (caserne Kléber) ainsi que les extensions tertiaires de la ville (Euralille).

Les enjeux concernent aujourd'hui le développement harmonieux de la ville tertiaire et de son patrimoine militaire, convoitant les mêmes espaces.

11 En se replongeant dans l'histoire, on peut noter que la première place forte de la ville, construite par Philippe le Bel, se fit sur ce front dans la partie surplombant le rivage 
commercial de la Basse Deûle (le château de Courtrai). Aujourd'hui démoli, il est toujours présent dans le tracé des voies de la rue des Tours placé au Levant de la ville, à l'opposé de la citadelle de Vauban qui plonge ses fondations dans les eaux du Couchant (fig. $\mathbf{n}^{\circ} \mathbf{2}$ ).

Figure 2

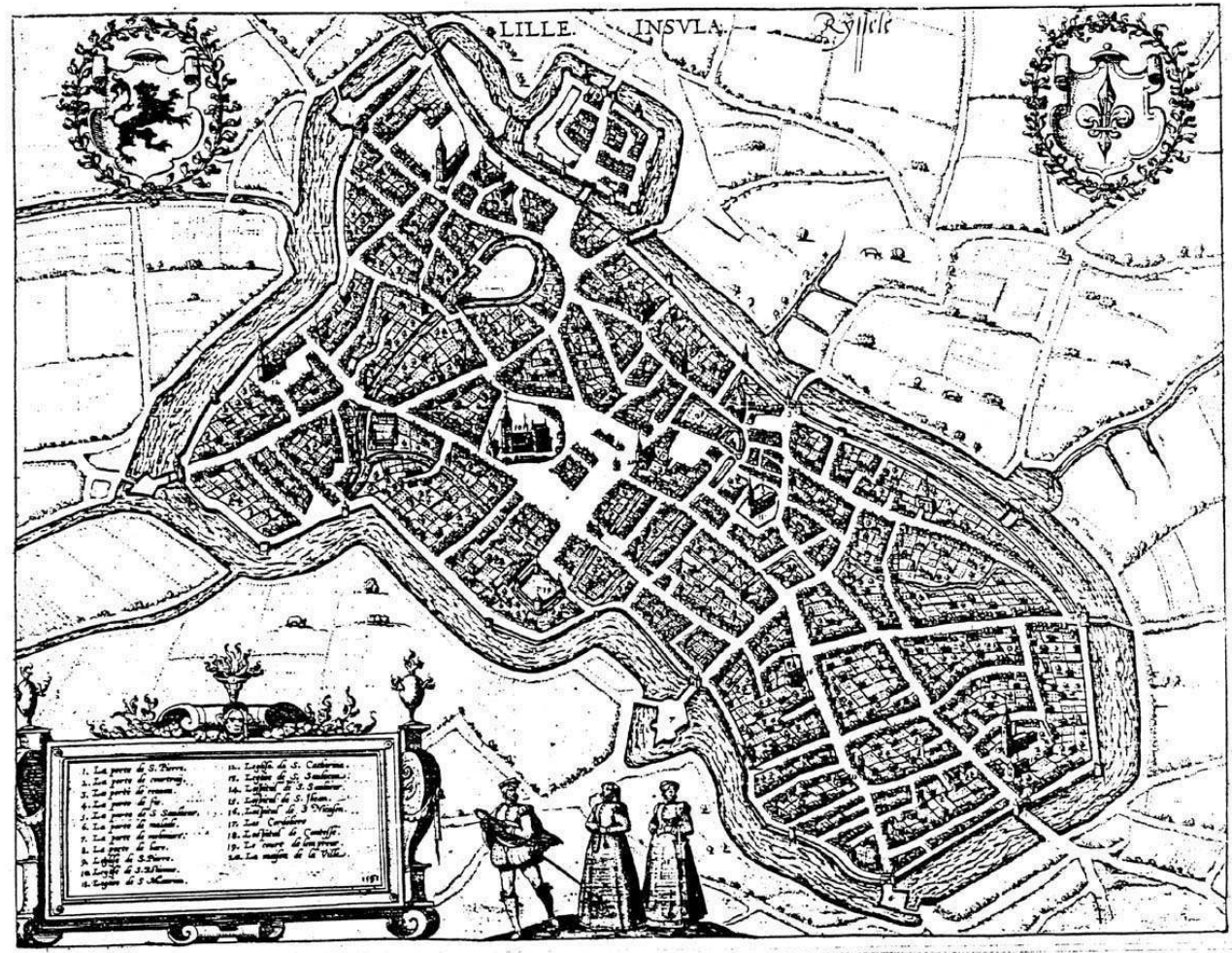

Le plan de Lille en 1581 (Braun). Le château de Courtrai commande le port de la Basse Deûle. Repro. E. Poncelet.

L'extension de la ville par les archiducs gouverneurs des Pays-Bas, au début du XVII ${ }^{e}$ siècle, a remonté l'enceinte urbaine de quelques centaines de mètres plus haut vers l'est.

Après la Paix des Dames et l'avènement de Charles-Quint en 1529, le Magistrat de Lille avait sollicité l'agrandissement de la ville. La richesse amenée par cette fragile époque de paix, favorisée sous l'âge d'or des archiducs Albert et Isabelle, gouverneurs des Pays-Bas, permit l'augmentation de la population. Lille possédait à l'époque 35000 habitants. Les faubourgs situés à l'ouest souhaitant s'insérer à la ville, il faudra attendre 1577 pour que les vieilles murailles de l'enceinte médiévale et du château de Courtrai soient démolies, permettant l'agrandissement de la ville.

En 1599, Isabelle, fille de Philippe II, épouse son cousin Albert, fils de l'Empereur d'Autriche, tous deux petits-enfants de Charles-Quint. Les archiducs gouverneurs des Pays-Bas font leur "joyeuse entrée » à Lille en 1600. Sous leur gouvernement sera construite l'enceinte espagnole de Lille.

15 Le projet d'extension de l'enceinte avait été préparé par Pierre Camp, ingénieur des archiducs, élargissant la ville de part et d'autre, vers l'est et vers l'ouest. En 1617, la nouvelle enceinte est construite au nord-est de la ville selon les principes des remparts bastionnés classiques généralisés dans les villes du Nord par les ingénieurs italiens de Charles-Quint (fig. $\left.\mathbf{n}^{\circ} \mathbf{3}\right)$. 


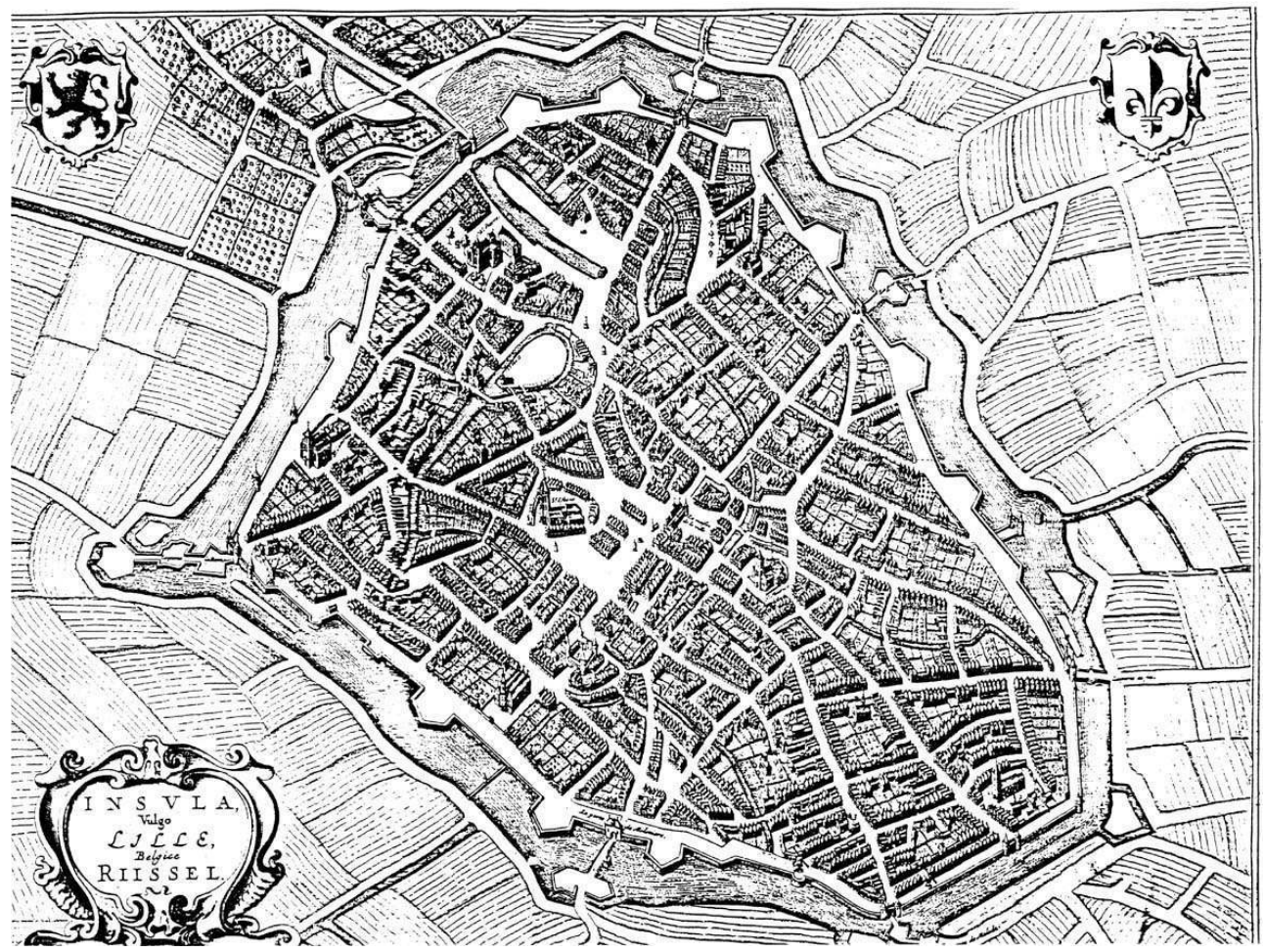

Le plan de Lille en 1645 (Boisseau). La ville est dans son état agrandi par l'enceinte espagnole. Les deux portes du front oriental possèdent des tenailles formant barbacane en avant des entrées. La porte Saint-Pierre possède une véritable barbacane précédant l'entrée.

Repro. E. Poncelet.

Ce front est fut percé de deux portes, la porte de Courtrai, devenue porte de la Madeleine puis porte de Gand, construite de 1621 à 1625 par Pierre Raoul, et la porte de Reneau devenue porte Saint-Maurice (appelée aujourd'hui porte de Roubaix), par Michel Watrelos et Jean Lesur. Ces ingénieurs-architectes étaient des maîtres-maçons de la région lilloise. Ils travaillaient sans doute sur des plans types imposés par le commandement espagnol. La ressemblance frappante entre les deux portes concerne le plan et les proportions. Les décors diffèrent cependant de manière importante, tant entre les faces intérieures et extérieures qu'entre les deux portes elles-mêmes (fig. $\mathbf{n}^{\circ} \mathbf{4}$ ). 
Figure 4
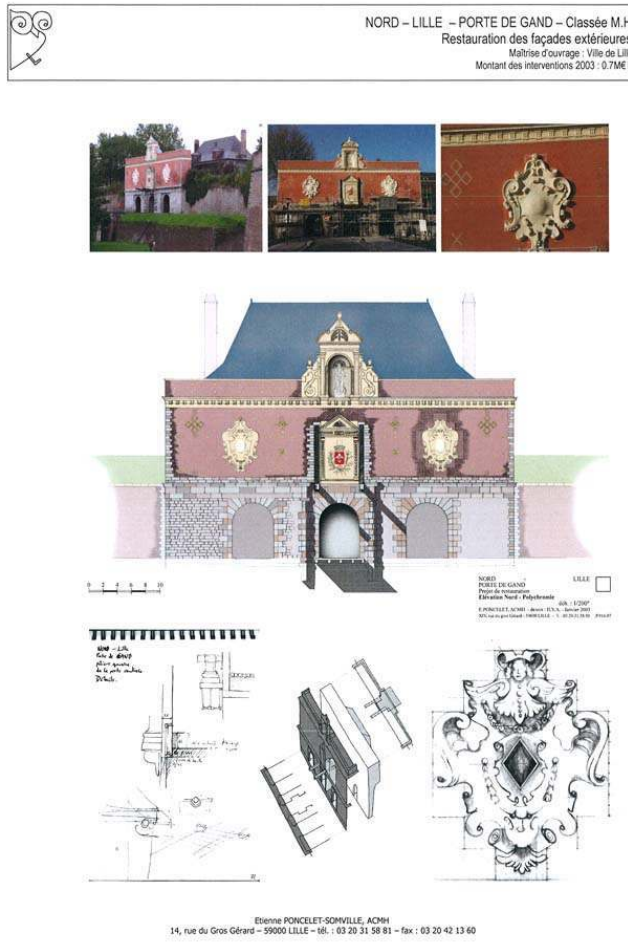

La restauration de la Porte de Gand.

(c) Agence Poncelet-Somville.

Les fronts des portes de Gand et de Roubaix témoignent encore de ces dispositions. Plus d'un demi-siècle avant Vauban, les architectes lillois nous livrent déjà l'image d'un front fortifié bastionné avec ses portes ornées de briques vernissées de couleur et de grands cartouches d'esprit Renaissance que nous avons restitués lors de la restauration faite en 2003. Ce chantier de restauration s'achève cette année par les aménagements des jardins qui nous ont permis de retrouver les systèmes de parapets des bastions et le jeu des rampes d'accès, de part et d'autre de la porte de Gand (fig. $\mathbf{n}^{\circ} 5$ ). 
Figure 5

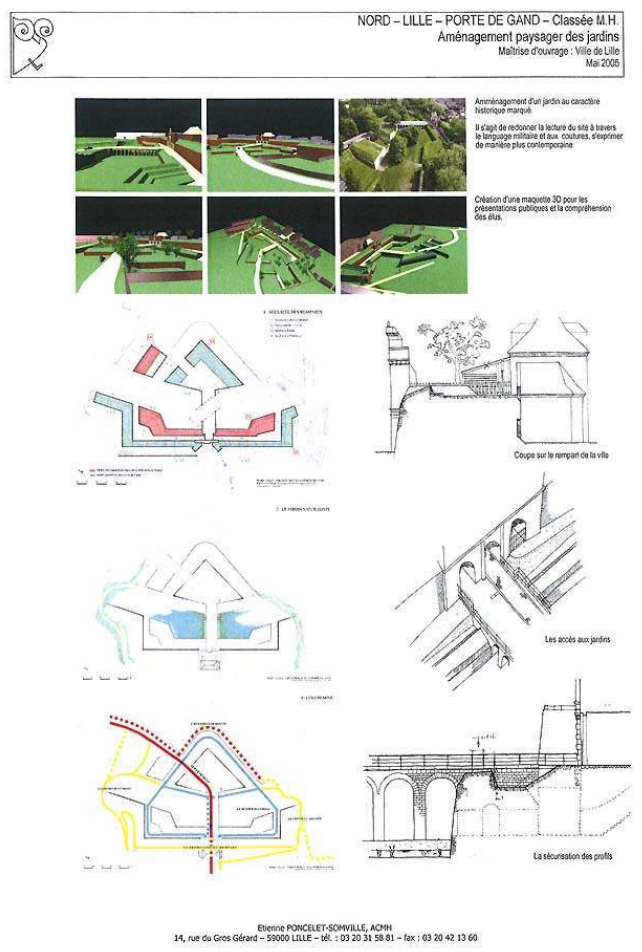

L'aménagement paysager des jardins de la porte de Gand.

(c) Agence Poncelet-Somville.

Ces vestiges de l'enceinte espagnole témoignent de la profonde imprégnation des cultures flamande, bourguignonne et espagnole mêlées au creux de nos Pays-Bas catholiques. Dans le dessin de leurs fortifications, ils auront servi de fondement aux ouvrages classiques dont Vauban viendra les couvrir cinquante ans plus tard. Dans leur expression architecturale, ils auront été une dernière floraison de l'esprit baroque de la Renaissance avant le classicisme français. Cette transition heureuse, c'est aux souverains espagnols, dignes héritiers des ducs de Bourgogne et des comtes de Flandres que nous le devons. Vauban viendra ensuite couvrir ce front d'un grand ouvrage à cornes (la corne de Gand) (fig. $\left.n^{\circ} 6\right)$ (fig. $\left.n^{\circ} 7\right)$. 
Figure 6

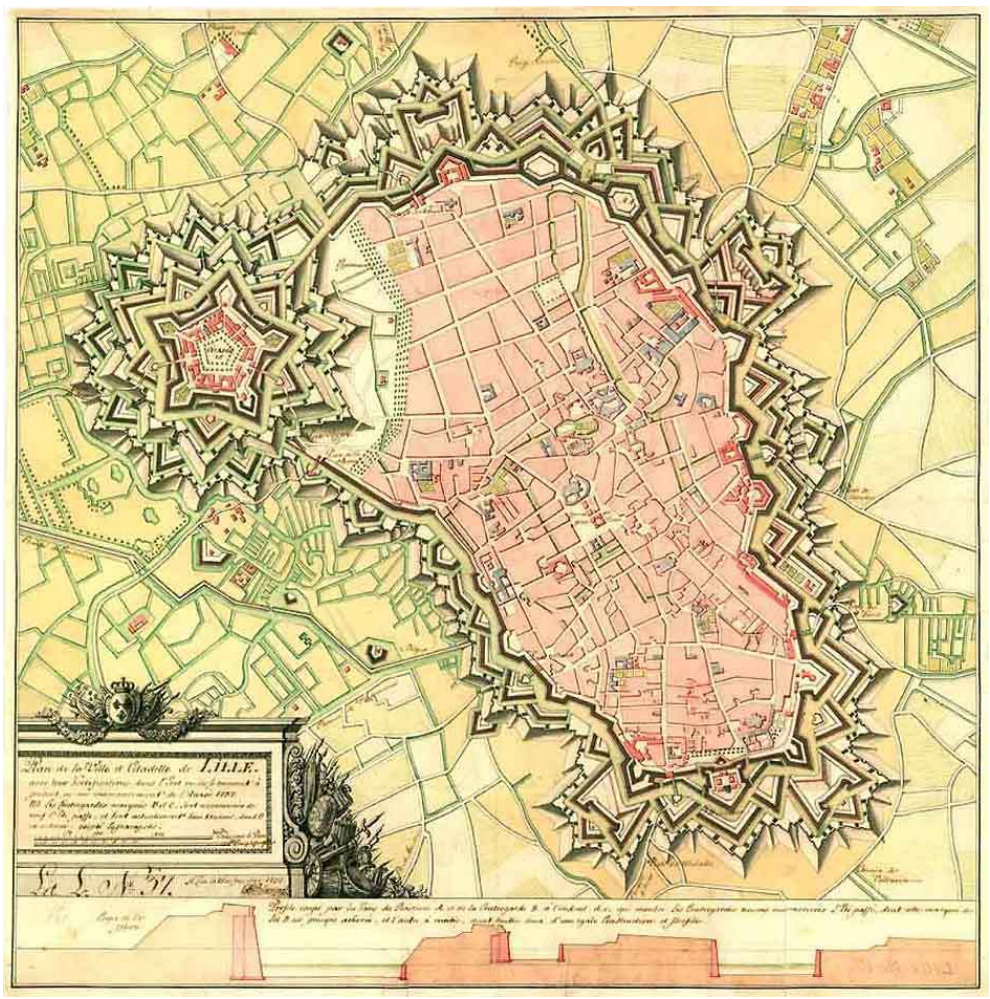

Le plan de Lille au XVIII e siècle. Le front oriental entre la porte d'eau au nord et le fort Saint-Sauveur, au sud, est couvert sur son front est par les ouvrages à cornes de Vauban.

Repro. E. Poncelet. 
Figure 7

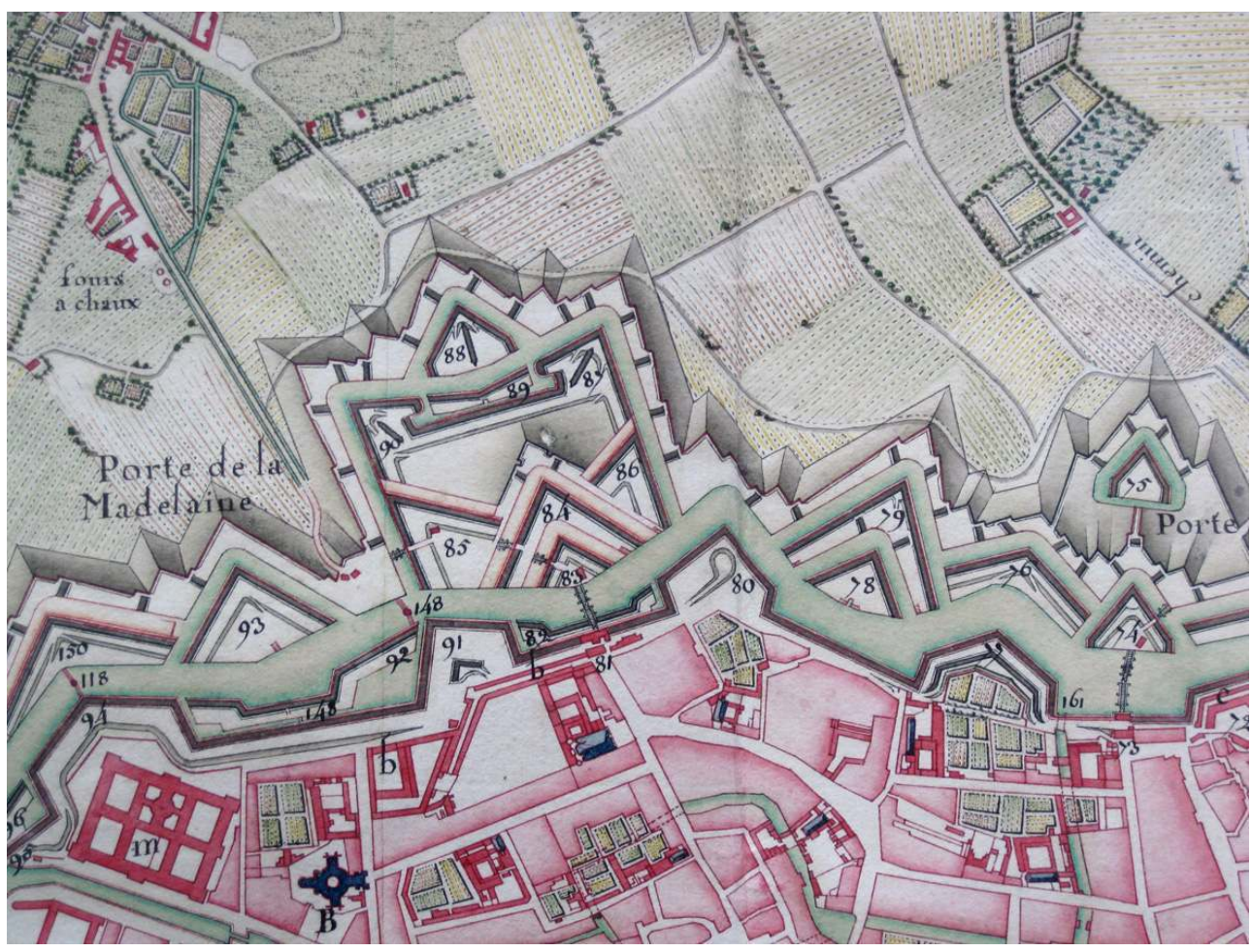

L'étude sur le projet de la Basse Deûle autour de l'ancienne porte d'eau.

(c) Agence Poncelet-Somville.

19 Au nord de ce front, le complexe de la porte d'eau témoigne du génie de Vauban dans l'un des ouvrages hydrauliques les plus complexes de la ville.

La porte d'eau de la Basse Deûle est protégée par un savant ouvrage à tenaillons épaulé par les bastions du Metz et du Rivage. Cet ouvrage, le long duquel transite la navigation, fonctionne un peu comme une barbacane d'eau (fig. $\mathbf{n}^{\circ} \mathbf{8}$ ). 


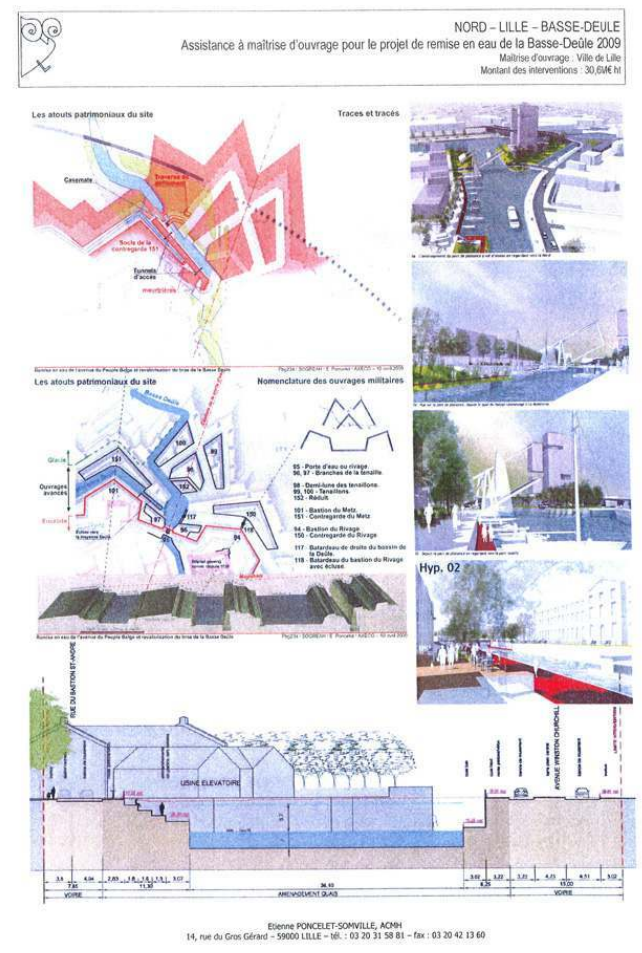

Le projet de remise en eau de la Basse Deûle.

(c) Agence Poncelet-Somville. de la citadelle, sans autre communication que le fossé nord, non navigable, creusé dans l' «île» géologique. Ce point haut du nord, sur l'éminence duquel on peut placer des moulins à vent, est protégé par l'ouvrage à corne du bastion Saint-André.

Au-delà des fortifications, la Basse Deûle rejoint la vallée de la Lys dans un cours marécageux exploité par la cense del Vacquerie placée sur sa motte entourée d'eau.

3 Le siège de 1708 va exploiter les faiblesses de cette zone complexe difficile à défendre. Les Alliés attaquent la ville à l'endroit précis de la porte d'eau, entre les deux ouvrages à corne de Saint-André et de la Madeleine. Les batteries de canons se placent sur les hauteurs de la rive droite de la Basse Deûle, fonctionnant comme un fossé de protection.

4 La porte d'eau de la Basse Deûle deviendra ensuite l'entrée du nouveau port de Lille, après le percement du nouveau canal de la Moyenne Deûle imaginé par Vauban mais réalisé seulement en 1750 .

5 La Moyenne Deûle ainsi créée est contrôlée par l'écluse de la Barre en amont et l'écluse de Saint-André près de la porte d'eau de la Basse Deûle. Celle-ci est protégée par la contregarde $151 \mathrm{du}$ bastion du Metz et sa double face. L'ancien batardeau 116 est démoli pour permettre la navigation.

Après de longs débats, les deux corps de bateliers de la Haute et de la Basse Deûle sont fusionnés en 1752. Le port de la Basse Deûle prend son essor, équipé d'une grue publique, le crasne pour " enlever les marchandises hors des bélandres ». 
27 La construction de la façade de l'hôpital général est commencée depuis 1739 et ne s'achèvera qu'un siècle plus tard.

L'époque de la Restauration voit l'apogée de ce système portuaire et défensif de la ville. La paix civile retrouvée marque le début de l'essor industriel de la ville. Le quai de la Basse Deûle, bordé par le nouveau palais de Justice, devient le centre économique et administratif de la ville. Le plan de 1829 montre que le cours de la Basse Deûle ne passe plus par les tenaillons mais longe la contregarde 151 pour traverser la face gauche de la lunette fonctionnant désormais comme une avant-porte d'eau.

29 Le plan d'eau compris entre celle-ci et l'ancienne porte d'eau forme une barbacane d'eau, sorte de souricière, fermement défendue par un chemin de ronde crénelé de meurtrières pour le tir rapproché.

30 L'état «Restauration » de ce site peut être considéré comme l'état de référence du plus grand développement de ce lieu. Les époques suivantes ne seront plus que l'expression d'une désaffectation progressive des lieux en terme d'activités jusqu'au projet d'aujourd'hui dans lequel, avec la Ville, nous proposons la remise en eau de la Basse Deûle.

31 De l'autre côté de la ville, à l'extrémité sud-est de ce front, se profile encore la Noble Tour, vestige du rempart bourguignon, insérée dans le bastion $n^{\circ} 68$, et aujourd'hui isolée au milieu d'un jardin du souvenir.

32 Le projet de réaménagement du quartier Saint-Sauveur sur lequel nous travaillons actuellement prévoit de relier à nouveau cet ouvrage à la promenade des remparts, depuis les vestiges de l'enceinte espagnole jusqu'au fort du Réduit, petit fortin construit par Vauban pour défendre le quartier sud de la ville, actuellement habité par le gouverneur militaire de Lille. Si l'on ajoute le front nord de la ville encore occupé par l'ouvrage à cornes de la caserne Saint-Ruth (ancien quartier Saint-André), la ville de Lille possède un continuum fortifié remarquable qui part de la citadelle, se poursuit sur le front nord et s'étire sur le front oriental jusqu'à la porte de Paris, devant l'entrée de l'hôtel de ville.

33 Cette promenade des remparts représente un parcours urbain historique et paysager exceptionnel, presque continu, que nous nous efforçons de faire revivre.

34 L'enjeu consiste aujourd'hui à faire de cet ensemble un véritable parc urbain faufilant ses courtines, bastions et profils engazonnés baignés par les cours d'eau, au cœur même d'une agglomération de près d'un million d'habitants.

\section{RÉSUMÉS}

De la porte d'eau de la Basse Deûle jusqu'au fort Saint-Sauveur, le front oriental de Lille, fortifié à l'époque espagnole, glisse ses courtines dans les entrelacs du périphérique et des gares. L'enjeu urbain actuel consiste à s'appuyer sur ces murs historiques pour " passer malgré tout " à travers cet écheveau urbain et retisser les fils de la continuité des promenades au cœur de la ville. Moins connus que le front occidental de la reine des citadelles, ces anciens espaces militaires sont une 
chance pour l'urbanisme de demain dont les opérations en cours de la Porte de Gand et de la Basse Deûle témoignent déjà.

The east wall, at Lille, fortified during the period of Spanish occupation, extends from the Porte d'Eau de la Basse-Deûle to the Saint-Sauveur fort. Its curtain walls emerge today in a landscape of ring roads and railway territories. The issue today is to profit from these historic walls in order to make some sense of the urban chaos and to reinstate some urban continuity in the city-centre walkways. Although they are not as well known as the western wall of this major fortified city, these former military properties are an exciting opportunity for tomorrow's town-planners, as the operations already underway at the Porte de Gand et de la Basse Deûle suggest.

\section{INDEX}

Mots-clés : enceinte espagnole, fortifications, Lille, patrimoine militaire

Keywords : fortifications, Lille, military heritage, Spanish surrounding wall

\section{AUTEUR}

\section{ÉTIENNE PONCELET}

Architecte en Chef des Monuments Historiques ponceletacmh@nordnet.fr 